\title{
Attenuation Characteristics and Time Delay of PD Electromagnetic Wave Propagation in GIS Systems
}

\author{
Tao Zhao, Member, IEEE, Martin D. Judd, Senior Member, IEEE and Brian G. Stewart, Member, \\ IEEE
}

\begin{abstract}
Modeling of electromagnetic wave propagation inside a gas-insulated switchgear structure can be helpful for understanding and improving the utilization of partial discharge detection by providing information to help determine more optimal positioning of ultra-high frequency sensors. This paper studies the effect of insulating spacers, $L$ - and T-type structures on electromagnetic wave propagation by simulating the time dependent wave propagation behavior for these arrangements within a gas-insulated switchgear busbar geometry. The attenuation characteristics and time delays produced by spacers, L- and T-type structures for partial discharge electromagnetic wave propagation behavior are presented. Emphasis is placed on comparison of attenuation characteristics and time delay between different observation points along the busbar both before and after the $90^{\circ}$ bends formed by $\mathrm{L}$ - and T-type structures. Moreover, a comparative analysis of the radial electric field at the gasinsulated switchgear tank and its rate of change with time is also conducted. The paper demonstrates that if the location of the ultra-high frequency sensor is chosen appropriately based on knowledge of the physical structures, the attenuation effects introduced by $L$ - and T-type structures could be reduced.
\end{abstract}

Index Terms-electromagnetic wave, propagation behavior, reflection effect, time delay, sensor location

\section{INTRODUCTION}

$\mathrm{P}$ ARTIAL discharge (PD) detection has become the most important method for assessing the insulation integrity and safety of gas-insulated switchgear (GIS) systems. The ultrahigh frequency (UHF) technique has been widely used for many years to detect PD in GIS systems, by which both PD detection and localization can be realized [1-3]. This technique is also much more practical for on-site application, being less sensitive to noise and more effective for PD localization compared with the conventional PD method according to IEC 60270. However, many factors act to prevent reaching high accuracy for PD detection and localization. First, a calibration to "apparent charge in $\mathrm{pC}$ " is not possible due to the complex PD pulse propagation characteristics, and second the PD detection sensitivity depends on various parameters such as barrier

Tao Zhao is with the Hebei Provincial Key Laboratory of Power Transmission Equipment Security Defense, North China Electric Power University, Baoding, 071003 China (e-mail: T.Zhao@ncepu.edu.cn).

Martin D. Judd is with the High Frequency Diagnostics and Engineering Ltd, Glasgow, G14 0BX, UK (e-mail: m.judd@hfde.co.uk). spacers, the geometry of the GIS system and the distance between any PD defect and the UHF sensor.

To solve the issues above, a technical brochure [4] published by CIGRE in 2016, collected available experience on PD sensitivity verification and described its practical applications for GIS. A detailed description of a two-step procedure is given in [4], and as a guideline, assists manufacturers and users in the effective application of the UHF PD method in GIS.

From a general point of view, GIS systems can be considered as having a coaxial waveguide geometry with a central internal high voltage (HV) conductor (or busbar) and grounded enclosure. In a practical installation, there are not only linear structures, but also many L-type and T-type structures.

Propagation characteristics of radiated PD electromagnetic (EM) waves in GIS systems are complicated. When an EM wave spreads in a GIS system, several phenomena may occur (such as reflection, refraction, resonance, wave mode conversion and attenuation), which combine to cause complex EM wave behavior [4-6].

Research and investigations have previously been conducted to explore the behavior of EM waves generated by PD inside GIS systems. Okabe et al. [7-9] investigated the reflection and mode conversion of EM waves in L-type and T-type GIS structures. Based on simulations in their High Frequency Structure Simulator, the propagation characteristics of multiple modes of an EM wave in GIS structures were illustrated. Hikita et al. [10-13] analyzed the EM attenuation trend through a spacer, an L-shaped branch, and a T-shaped branch by means of both simulation and experiments. They concluded that the attenuation trend was mainly attributed to superimposition of the transverse electric (TE) and transverse electric and magnetic (TEM) modes. Behrmann et al. [14-16] explored EM wave behavior inside GIS by performing radio frequency (RF) network analyzer measurements and 3D finite-element modeling. Their results showed that refinements to finite element modelling (FEM) techniques result in a closer match to actual measurements. Plentiful relevant research on EM wave behavior in various GIS structures has obtained some meaningful results and available experiences. However, due to the complexity of the EM wave behavior in GIS systems, there

\footnotetext{
Brian G. Stewart is with the Institute of Energy and Environment, University of Strathclyde, Glasgow, G1 1XW, UK (e-mail: brian.stewart.100@strath.ac.uk).
} 
remain issues that require further study, including determining the most suitable positions to place UHF PD sensors for optimum signal sensitivity [17]. A further important topic relates to the precise nature of propagation delay through various internal constructions, which is important for implementing accurate PD localization based on time differences between PD signals measured at different UHF sensors.

This paper applies 3D finite element modeling for time dependent simulation investigations to gain an improved understanding of the propagation behavior of EM waves in GIS systems. The study focuses on the influence of barrier spacers, L-type and T-type structures on signal attenuation and time delay. By using point probes to monitor the UHF electric field at various positions on the GIS tank, optimal positions for sensor installation can be deduced. The main contribution of this paper is to demonstrate that the attenuation effects introduced by L-type and T-type structures can be reduced if the placement of UHF sensors is defined appropriately based on knowledge of the physical structures. In addition, an improved understanding of UHF propagation time delay for these structures can assist with determining any time adjustments necessary to improve the accuracy of PD source localization.

\section{Simulation Model}

The simulation model of the linear, L-type and T-type GIS structures used in this study are constructed using COMSOL Multiphysics software. Diameters of the inner and enclosure conductors typical of $400 \mathrm{kV}$ GIS were defined as $125 \mathrm{~mm}$ and $420 \mathrm{~mm}$, respectively. Both are modeled as perfect electric conductors (PEC). Sulfur hexafluoride $\left(\mathrm{SF}_{6}\right)$ is the insulation gas considered (relative permittivity $\varepsilon_{r}=1$, relative permeability $\mu_{r}=1$, and electrical conductivity $\sigma=0$ ). Dielectric spacers are considered as epoxy resin $\left(\varepsilon_{r}=4, \mu_{r}=1\right.$ and $\left.\sigma=0\right)$. The field formulation used for the time-domain simulations is described by the following equations [16]:

$$
\begin{gathered}
\nabla \times\left(\frac{1}{\mu_{r}} \nabla \times \mathbf{A}\right)+\mu_{0} \sigma \frac{\partial \mathbf{A}}{\partial t}+\mu_{0} \varepsilon_{0} \frac{\partial}{\partial t}\left(\varepsilon_{r} \frac{\partial \mathbf{A}}{\partial t}\right)=0 \\
\mathbf{n} \times \mathbf{E}=0 \text { (PEC boundary condition) } \\
Z_{\text {port }}=\frac{U_{\text {port }}}{I_{\text {port }}} \text { (Lumped port with voltage input) } \\
\mathbf{A}(\mathbf{r}, 0)=0 \text { (Zero initial condition) }
\end{gathered}
$$

where $\mathbf{A}$ is the magnetic vector potential, $\mu_{0}$ represents the magnetic permeability of vacuum, $\mu_{r}$ is the relative magnetic permeability, $\varepsilon_{0}$ is the electric permittivity of vacuum, $\varepsilon_{r}$ refers to the relative permittivity of the propagation medium, $\mathbf{n}$ is a unit vector normal to the surface and $Z_{p o r t}$ is the wave impedance of the lumped (input) port. The lumped port node is used to apply a voltage or current excitation of a model in COMSOL Multiphysics. The output port is set to a scatter boundary for modeling an open boundary and this boundary is transparent for incoming plane waves with any angle of incidence. Therefore, there is no reflection back into the structure at the output port during the simulation.

A common approach in the numerical simulation of electromagnetic waves generated by PD is to approximate the pulse as having a Gaussian shape in the time domain $[3,11,13]$.
An advantage of using a Gaussian pulse in computational electromagnetic simulations is that its time-derivatives do not contain discontinuities that can lead to instability in the iterative numerical calculations. To represent a PD pulse, the Gaussian pulse [18-19] shown in Fig. 1 with half amplitude width of 0.3 ns is used. This was applied to the coaxial structure of the GIS at the input plane of the GIS busbar. Because of the stochastic nature of PD, pulse amplitude is usually quite variable in practice. Hence, for the purpose of simulation, the model uses a normalized PD pulse amplitude and the electric field results are similarly presented on a normalized amplitude scale.

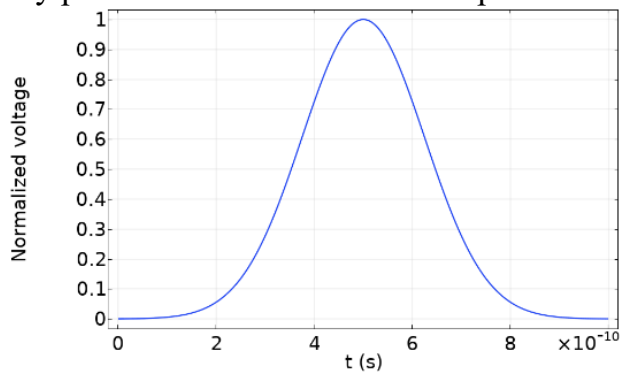

Fig. 1. The Gaussian pulse at the source port.

\section{SimULATION RESUlts}

\section{A. Linear Structure}

The model setup, schematically shown in Fig. 2, is a section of a gas-filled coaxial chamber. The symmetry of the structure allows for a 2D axisymmetric model geometry. There are 3 barrier spacers in the linear structure placed at equal intervals of 1 meter and the thickness of spacers is $30 \mathrm{~mm}$. Point probes that record the evolution of electric field values during the simulation are set with positions shown as red points in Fig. 2. The coordinates of these point probes are separated at equally spaced intervals along the principal forward propagation $z$ direction of the geometry chamber. Time dependent plots of the electric field in the radial $r$-direction during a time range covering $[0,0.1 \ldots 20 \mathrm{~ns}]$ are produced once the simulation is completed.

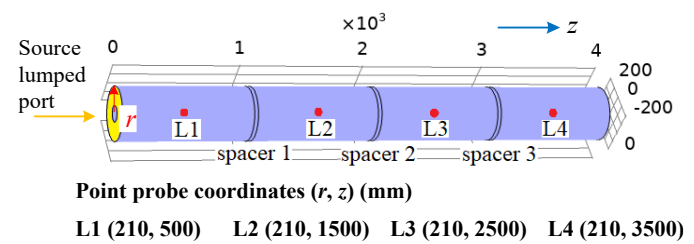

Fig. 2. Linear structure and coordinates of the point probes.

Fig. 3 shows the time-varying electric field at the point probes in the linear structure. There exist four larger positive electric field peaks which reveal the main forward-travelling EM wave passing the four point probe positions. In addition, there are many peaks with lower values, which are generated by reflection from the spacers.

In the modeling, after passing through one spacer, the amplitude of the electric field declines by about $6 \%$. According to the Fresnel Equation, the attenuation level during EM wave reflection and refraction is related to the angle of incidence and the dielectric constant of the materials on both sides of the interface. As the dielectric constant of the spacer materials increases, so does the level of energy reflected.

The time delay effect of the EM wave propagation is also 
evident. The distance between L1 and L4 is $3000 \mathrm{~mm}$. The time-of-arrival (TOA) result obtained from the time interval between the largest value peaks of L1 and L4 in Fig. 3 is approximately $10.3 \mathrm{~ns}$ with three barrier spacers installed in the model. Without the barrier spacers installed, the TOA is about 10 ns. This indicates that one barrier spacer in the model produces a time delay of around $0.1 \mathrm{~ns}$ that would give rise to a localization error of approximately $3 \mathrm{~cm}$ provided that the velocity of EM wave propagation approximates to $30 \mathrm{~cm} / \mathrm{ns}$. The result of the time delay produced by the spacer in this model is consistent with that of reference [16] in which the result is $0.16 \mathrm{~ns}$ from practical TOA measurement with the spacer material having a relative electric permittivity of 4.2.

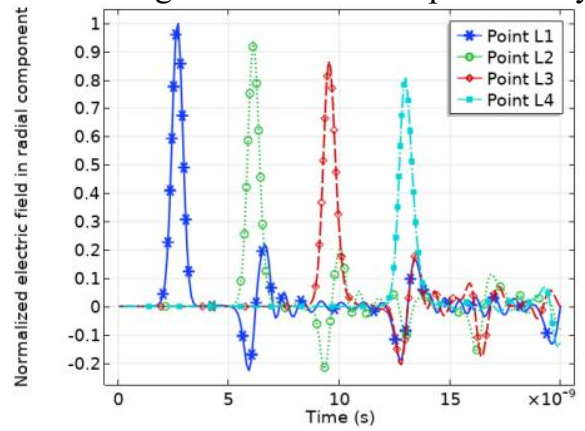

Fig. 3. Normalized electric field in radial direction versus time (the maximum on point $\mathrm{L} 1$ is the normalized field benchmark).

\section{B. L-type Structure}

The geometry of the L-type GIS structure used in this study is schematically shown in Fig. 4. The parameters of the dielectric materials and the diameters of the inner and enclosure conductor are the same as those of the linear structure. Four point probe positions (A, B, C, and D) and their coordinates are shown as red points in Fig. 4.

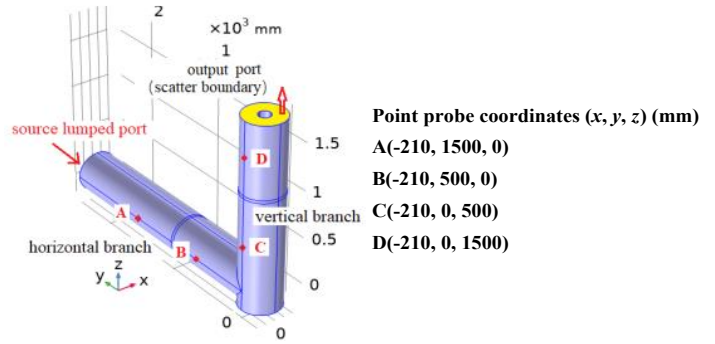

Fig. 4. Geometry of L-type structure and coordinates of point probes.

Fig. 5 reveals the time-varying electric field at the point probe positions on the horizontal branch and vertical branch of the L-type structure. The propagation and reflection of EM waves are also illustrated in Fig. 5. When the EM wave passes each point probe, there is a peak in the field curve. Subsequently, the curves start to fluctuate over time and there are many peaks with lower amplitudes generated by the reflections from the spacers and Lbend. Fig. 5a shows that before passing through the L-bend, the propagation and reflection of EM waves are similar to those in the linear structure. However, Fig. 5b shows significantly different characteristics when the EM wave propagates into the vertical branch. The attenuation rate of the amplitude is nearly $50 \%$ which is much larger than that of the spacer.

Fig. 6 displays a snapshot at $t=11.2 \mathrm{~ns}$ and shows the electric field distribution as the EM wave passes through the L-bend. When the wave propagates into the vertical branch, its waveform and amplitude change significantly, which indicates overlap of adjacent waves caused by reflections within the L-bend. Furthermore, the electric field distribution in Fig. 5 and Fig. 6 indicates that the electric field properties differ quite significantly for different positions. Thus, for electric field detection, the outputs of UHF PD sensors can be quite different for different sensor installation locations. In a cross section of a GIS cylinder or pipe there are generally two orientations, horizontal (0 degree) and vertical (90 degree) used in the mounting of UHF sensors. That is to say, they are usually located either on the sides of the GIS tank, or the top/bottom of the tank. In order to compare the sensitivity in the horizontal and vertical planes, it is necessary to obtain the evolution of electric field at these most common sensor mounting positions. To investigate this further, point probes are positioned at locations P1 - P5 around the L-bend (see Fig. 7a and Table I for the specific coordinates). P1 and P2 are positioned before the L-bend, while P3, P4, and P5 are positioned in the same horizontal plane after the L-bend. P4 is positioned where the path length around the bend is smallest whilst P5 is positioned where the bend path length is largest. P3 is the intermediary bend position. Note that these locations are commonly used for PD UHF sensor installations $[8$, $15]$.

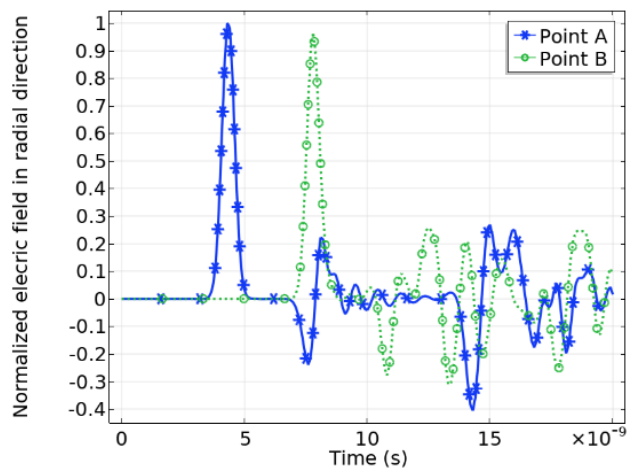

(a)

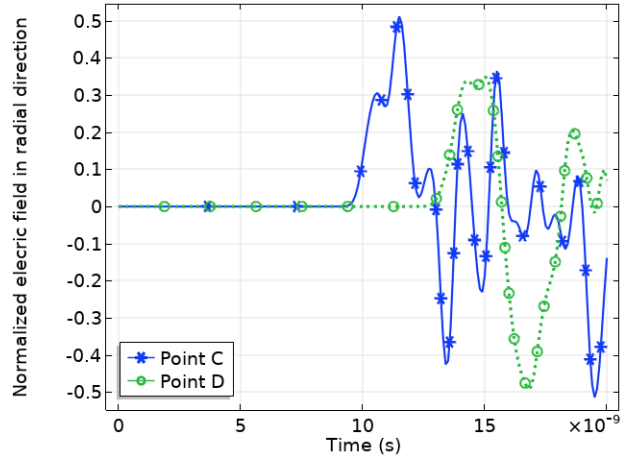

(b)

Fig. 5. Electric field in radial direction versus time (the maximum on point $A$ is as the normalized field benchmark). (a) Point probes A and B on horizontal branch. (b) Point probes $\mathrm{C}$ and $\mathrm{D}$ on vertical branch.q

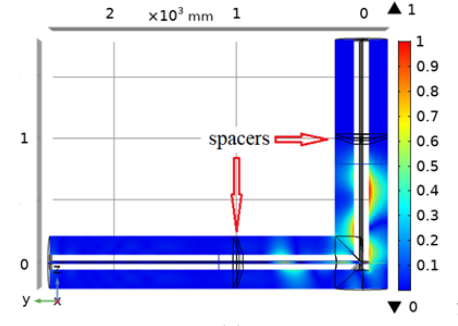

(a)

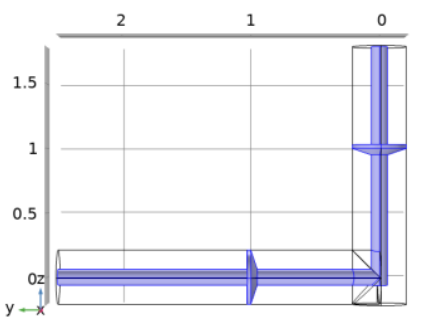

(b)
Fig. 6. Electric field distribution in L-type structure at $t=11.2$ ns. (a) Electric field distribution. (b) Profile of inner conductor and spacers of L-type structure. 
Fig. $7 \mathrm{~b}$ and $7 \mathrm{c}$ and Table I compare the difference of the electric field for these positions. As expected, P1 and P2 produce very similar results as the signal propagates. The smaller reflected signals due to the L-type bend produce small variations in the P1 and P2 signals based on slightly different L-bend reflection interferences for the signals at these measurement positions.

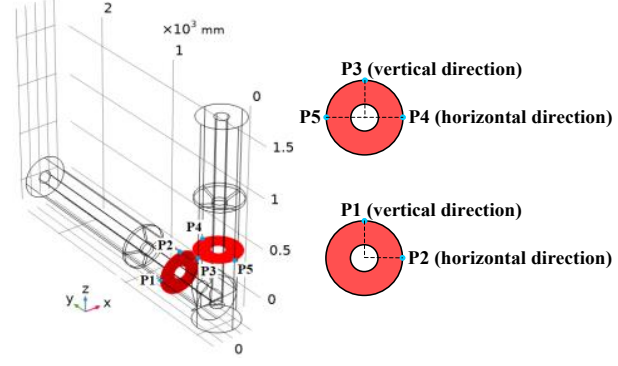

(a)

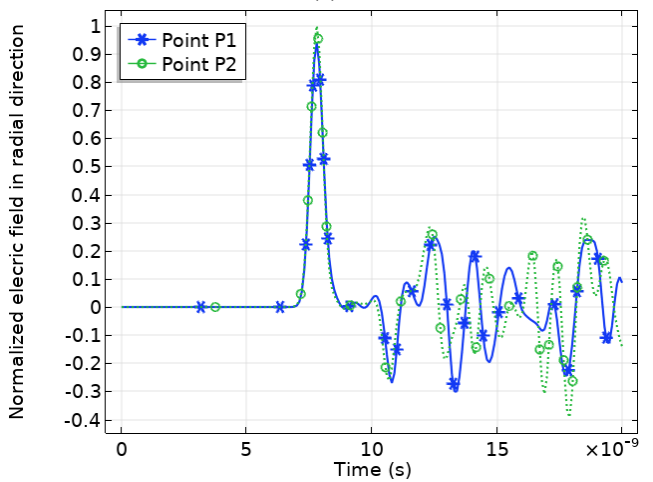

(b)

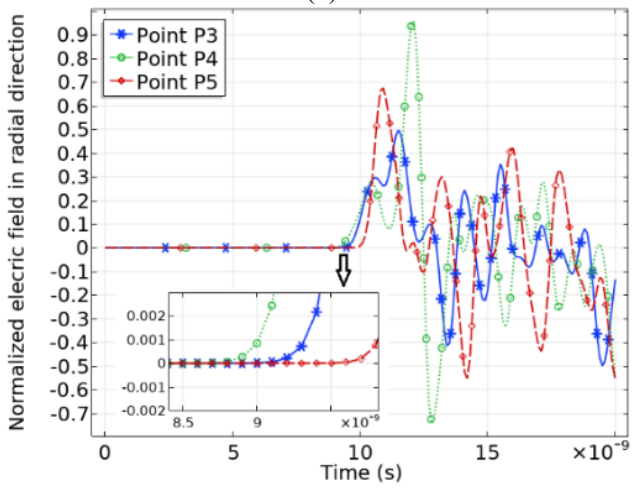

(c)

Fig. 7. Electric field in radial direction at point probes (The maximum at $\mathrm{P} 2$ is as the normalized field benchmark). (a) Test point positions. (b) Point probes P1 and P2 on horizontal branch. (c) Point probes P3, P4, and P5 on vertical branch.

In Table I, the maximum difference of the peak values at the test point locations $\mathrm{P} 3, \mathrm{P} 4$, and $\mathrm{P} 5$ ranges up to $47 \%$. It is noted that the attenuation at test point $\mathrm{P} 3$ is the most significant; the detection sensitivity is higher at the other two test points $\mathrm{P} 4$ and P5, with P4 being the highest and just lower than the peak values from P1 and $\mathrm{P} 2$. Thus, if the location of the sensor is chosen appropriately as $\mathrm{P} 4$, the measurement sensitivity will almost be unaffected by the L-type structure [17].

The time delay introduced by the L-type structure is also presented in Fig. 7c and Table I for the UHF signal arrival time at points P3, P4 and P5. Based on the point of signal departure from zero in the simulated signals, the largest difference between the TOA occurs as expected between P4 and P5. The signal arrives at P5 $0.9 \mathrm{~ns}$ later, which corresponds to a distance of $27 \mathrm{~cm}$ for wave propagation. Overall, the changes to wave propagation caused by the L-type structure are much more severe than those caused by the spacer. This distortion may also decrease the signal-to-noise ratio (SNR) of the measured signals, which could in turn influence the TOA measurement accuracy. Considering a more practical thresholding approach to determining TOA, if $5 \%$ of the P2 signal peak value is used for arrival time estimation, the results obtained are also shown in Table I. The largest difference between TOA occurs between P4 and P5 and is about $0.7 \mathrm{~ns}$, corresponding to a distance of about $21 \mathrm{~cm}$ for wave propagation.

TOA values will change slowly if another threshold is selected because the amplitude of the waveforms varies over time from place to place and the peak values are also different for different test positions. Comparison of different thresholds of $5 \%, 8 \%$ and $10 \%$ at point probes $\mathrm{P} 3, \mathrm{P} 4$ and $\mathrm{P} 5$ are also presented in Table I.

TABLE I

ATTENUATION AND TIME DELAY EFFECT OF L-BEND.

\begin{tabular}{l|c|c|c|c|c}
\hline $\begin{array}{c}\text { Point probe } \\
\text { positions } \\
(x, y, z) \\
(\mathrm{mm})\end{array}$ & $\begin{array}{c}\text { Normalized } \\
\text { electric field } \\
\text { in radial } \\
\text { direction }\end{array}$ & $\begin{array}{c}\text { TOA based } \\
\text { on signal } \\
\text { departure } \\
\text { from zero } \\
(\mathrm{ns})\end{array}$ & $\begin{array}{c}\text { TOA based } \\
\text { on 5\% } \\
\text { threshold } \\
\text { of peak } \\
(\mathrm{ns})\end{array}$ & $\begin{array}{c}\text { TOA based } \\
\text { on } 8 \% \\
\text { treshold } \\
\text { of peak } \\
(\mathrm{ns})\end{array}$ & $\begin{array}{c}\text { TOA based } \\
\text { on } 10 \% \\
\text { threshold } \\
\text { of peak } \\
(\mathrm{ns})\end{array}$ \\
\hline $\begin{array}{l}\text { P1 (-210, } \\
500,0)\end{array}$ & $98 \%$ & 6.6 & 7.2 & 7.2 & 7.3 \\
$\begin{array}{l}\text { P2 (0, 500, } \\
210)\end{array}$ & $100 \%$ & 6.6 & 7.2 & 7.2 & 7.3 \\
P3 (-210, 0, & $51 \%$ & 9.0 & 9.8 & 9.9 & 9.9 \\
$500)$ & $98 \%$ & 8.7 & 9.5 & 9.7 & 9.8 \\
$\begin{array}{l}\text { P4 (0, 210, } \\
500)\end{array}$ & $69 \%$ & 9.6 & 10.2 & 10.3 & 10.3 \\
P5 (0,-210, \\
$500)$
\end{tabular}

\section{T-type Structure}

The geometry of the T-type GIS structure is illustrated in Fig. 8. There are two cases of the T-type structure based on the location of the source port, i.e. Case I and Case II. Point probes $\mathrm{A}, \mathrm{B}, \mathrm{C}, \mathrm{D}$, and $\mathrm{E}$ are set with positions shown as red points in Fig. 8.

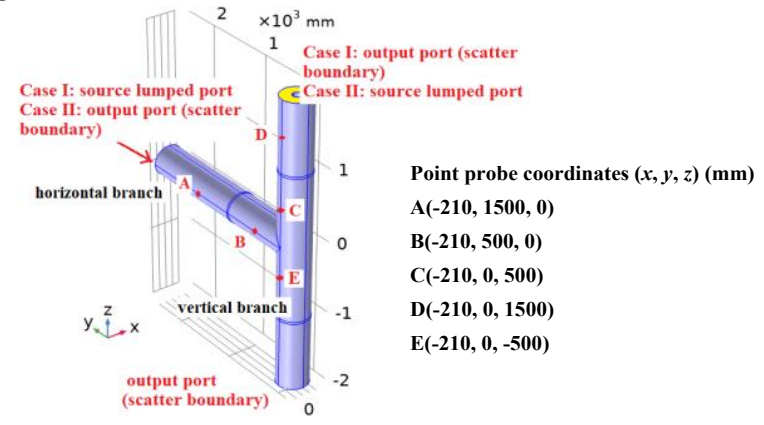

Fig. 8. Geometry of T-type structure and coordinates of point probes.

\section{1) Case I}

For Case I, the signal port is into the horizontal branch of the geometry (see Fig. 8). For the $x-y$ plane at $z=0$, the propagation path of the EM wave in Case I is symmetrical. Fig. 9 presents the time-varying electric field at the point probes on both the horizontal and vertical branches. Similar to the results of the Ltype structure, different signal characteristics at each position are evident when the EM wave propagates into the vertical branch of the T-type structure. The electric field in Fig. 9b 
indicates overlap of adjacent wave peaks.

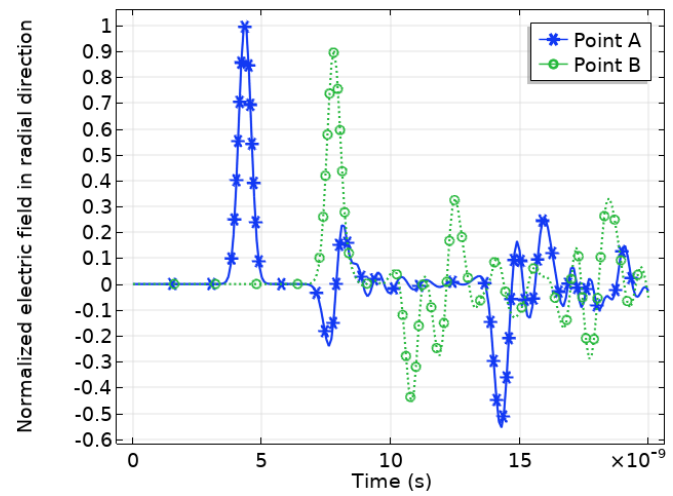

(a)

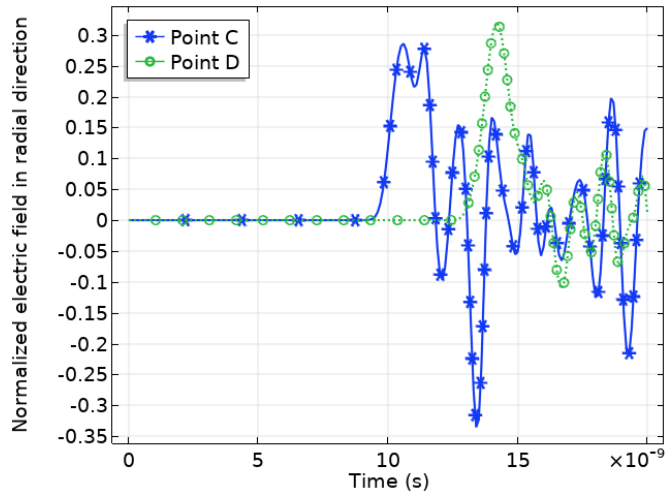

(b)

Fig. 9. Electric field in radial direction versus time at point probes (the maximum on point $\mathrm{A}$ is the normalized field benchmark). (a) Point probes $\mathrm{A}$ and $\mathrm{B}$ on horizontal branch. (b) Point probes $\mathrm{C}$ and $\mathrm{D}$ on vertical branch.

Differences in the electric fields for positions P1-P5 are compared in Fig. 10 and Table II. The positions are similar to the L-bend positions analyzed in Fig. 7a. In Table II, the peak values at locations $\mathrm{P} 3, \mathrm{P} 4$, and $\mathrm{P} 5$ are $37 \%, 63 \%$, and $73 \%$ respectively. Therefore, for electric field detection, if the EM wave signal passes through the T-type structure and is detected by a sensor, the installation position of the sensor significantly influences the strength of the received signal. By comparison, a result similar to the L-type situation is also obtained. The most significant attenuation takes place at test point P3. Detection sensitivity is higher at the other two test points $\mathrm{P} 4$ and P5 in the horizontal plane of the T-bend (defining the tank to be the horizontal direction). Thus, if the location of the sensor is chosen appropriately, i.e. P5, the effect of the attenuation by the T-type structure can be minimized [17].

Time delay caused by the T-type structure is shown in Fig. 10c and Table II. The largest difference between TOA of the three point probes $\mathrm{P} 3, \mathrm{P} 4$, and $\mathrm{P} 5$ is $0.9 \mathrm{~ns}$ based on the point of signal departure from zero and $0.7 \mathrm{~ns}$ based on the $5 \%$ threshold, which is similar to that of the L-type structure.

TABLE II

ATTENUATION AND TIME DELAY EFFECT OF T-BEND (CASE I).

\begin{tabular}{l|c|c|c}
\hline $\begin{array}{c}\text { Point probe } \\
\text { positions }(x, y, z) \\
(\mathrm{mm})\end{array}$ & $\begin{array}{c}\text { Normalized } \\
\text { electric field in } \\
\text { radial direction }\end{array}$ & $\begin{array}{c}\text { TOA based on } \\
\text { signal departure } \\
\text { from zero }(\mathrm{ns})\end{array}$ & $\begin{array}{c}\text { TOA based on } \\
5 \% \text { threshold } \\
\text { of peak (ns) }\end{array}$ \\
\hline P1 $(-210,500,0)$ & $99 \%$ & 6.5 & 7.1 \\
P2 $(0,500,210)$ & $100 \%$ & 6.5 & 7.1 \\
P3 $(-210,0,500)$ & $37 \%$ & 8.9 & 9.8 \\
P4 $(0,210,500)$ & $63 \%$ & 8.5 & 9.5 \\
P5 $(0,-210,500)$ & $73 \%$ & 9.4 & 10.2 \\
\hline
\end{tabular}

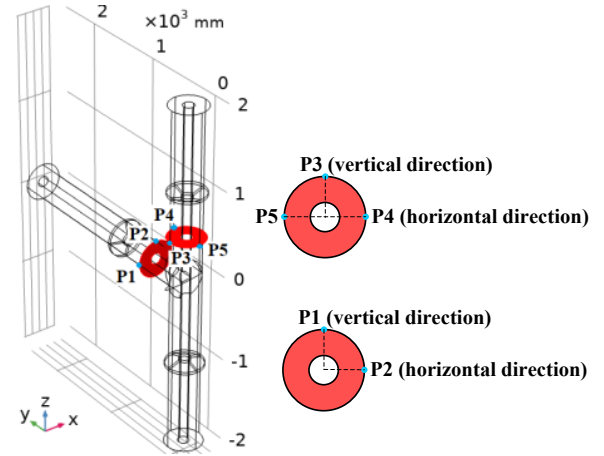

(a)

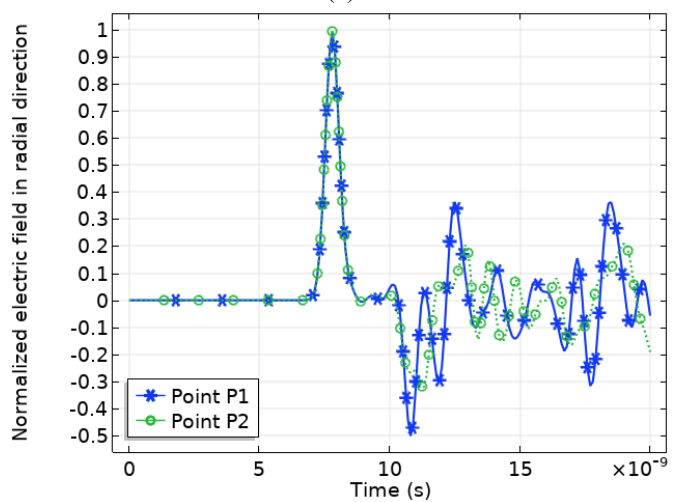

(b)

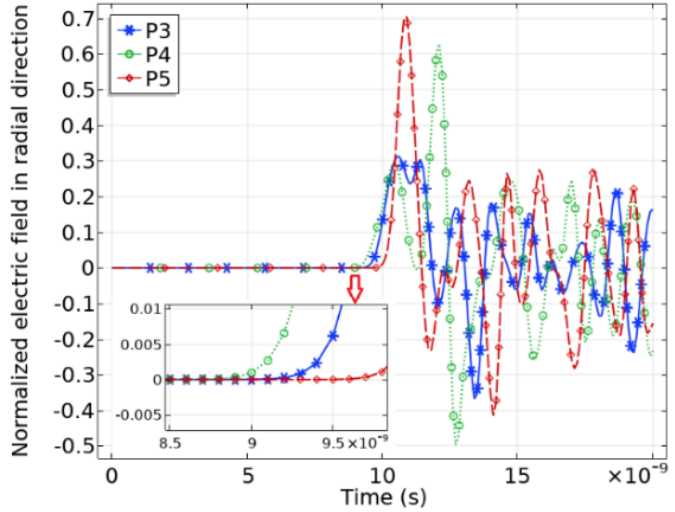

(c)

Fig. 10. Comparison of electric field at point probes (the maximum on point P2 is normalized field benchmark). (a) Coordinates of the point probes. (b) Point probes P1 and P2 on horizontal branch. (c) Point probes P3, P4, and P5 on vertical branch.

\section{2) Case II}

Fig. 11 shows the electric field in the radial direction at the point probe positions. It can be seen that the attenuation characteristics in the tangential and forward directions are different. The peak value of the wave reaching the forward direction test point $\mathrm{E}$ is about twice as large as the signal measured at test point $\mathrm{B}$. The TOA at test point $B$ is $0.9 \mathrm{~ns}$ earlier than that at test point $\mathrm{E}$ based on the point of signal departure from zero and $0.7 \mathrm{~ns}$ earlier based on a $5 \%$ threshold.

It is evident that the attenuation characteristics and the TOA vary with different positions. To clarify the attenuation characteristics and the time delay produced by the T-bend, a detailed comparison is conducted at more point probe positions i.e. P1, P2, .. P6. The locations of the point probes are shown in Fig. 12 and the comparisons are presented in Fig. 13 and Table III. 


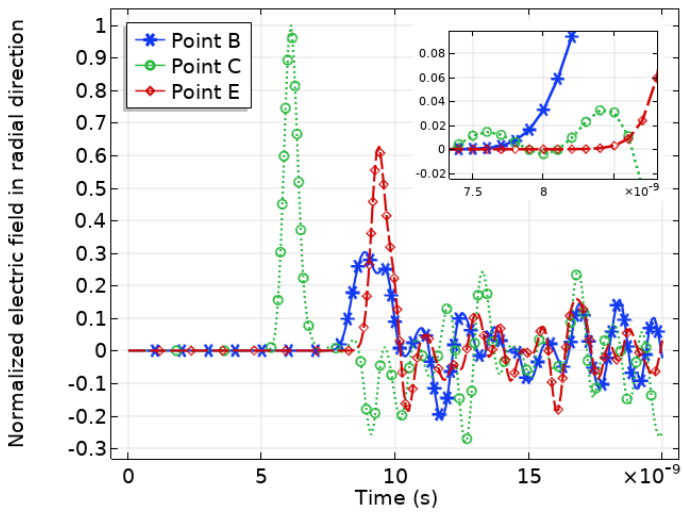

Fig. 11. Electric field in radial direction versus time (the maximum on point $\mathrm{C}$ is as the normalized benchmark).

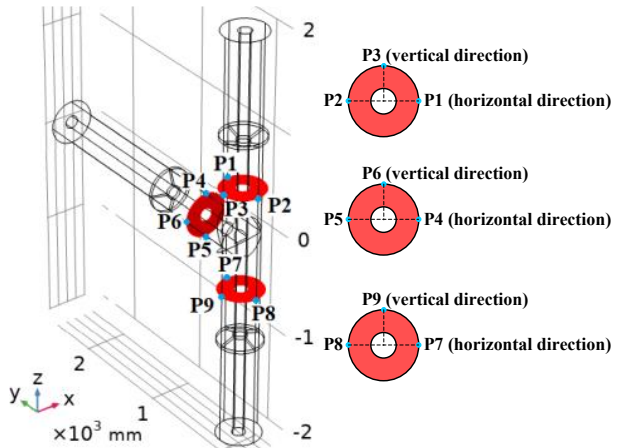

Fig. 12. Locations of the point probes.

Fig. 13a shows the electric field at three test points P1, P2, and $\mathrm{P} 3$ before the T-bend, which are located in the same transverse plane of the wave propagation direction. It illustrates that amplitudes and arrival times of the EM wave remain the same at the three test points. Fig. 13b shows the electric field at three test points P4, P5, and P6 in the T-bend tangential direction, and which are located in the same transverse plane. As can be seen from Fig. 13b, significant changes have taken place both in amplitude and TOA between these three points (see Table III for their specific positions). Fig. 13c displays the electric field at three test points P7, P8, and P9 in the forward direction of the tube geometry, which are located in the same transverse plane of the wave propagation direction. It shows that TOA of the EM wave stays almost the same for the three points. However, there exist small differences between signal amplitudes as can be seen in Table III.

Table III compares the difference of the electric field attenuation for all point probes. The attenuation at the points in the tangential direction is much higher than that in the forward direction. Furthermore, the amplitudes of electric field among the points in the tangential direction also differ greatly and the attenuation is more severe than in the Case I T-type structure.

Thus, for PD detection sensitivity in the T-type structure in GIS, the optimal position for sensor installation is in the forward branch where the sensor can have the highest sensitivity, the second-best position is at the $\mathrm{T}$ branch of Case $\mathrm{I}$, and the worst position is at the $\mathrm{T}$ branch of Case II. Additionally, the detection sensitivity is higher at the test points in the horizontal plane compared to those positions in the vertical plane of the cross section of the tank (defining the tank to be the horizontal direction). Therefore, if the location of the sensor is chosen appropriately, as described above, the measurement sensitivity obtained can reduce the attenuation caused by the T-type structure.

A comparison of TOA for all point probes is also shown in Table III. TOA at the points in the tangential direction vary greatly, while the values at the points in forward direction are essentially the same. Furthermore, the TOA of the points in the forward direction is later than that in the tangential branch and the median value of time delay is about $0.9 \mathrm{~ns}$ based on signal zero-crossing and $0.7 \mathrm{~ns}$ based on $5 \%$ peak value. Thus for TOA estimation, sensors installed in the forward branch will provide more consistent results, but there will be a certain time delay compared to sensors installed in the tangential direction.

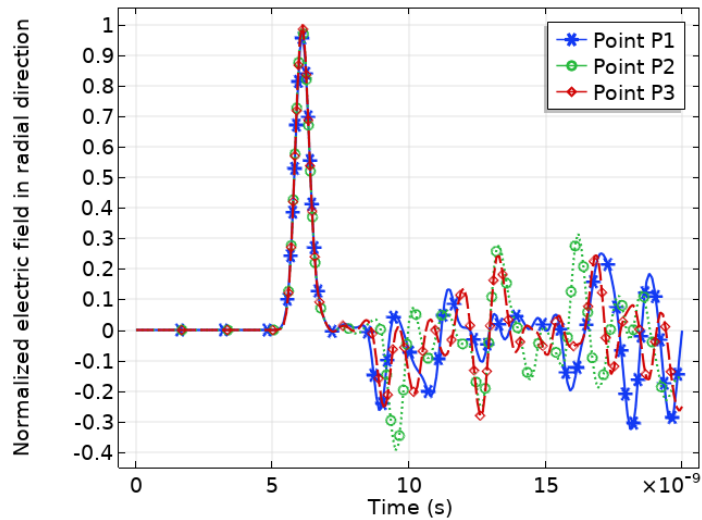

(a)

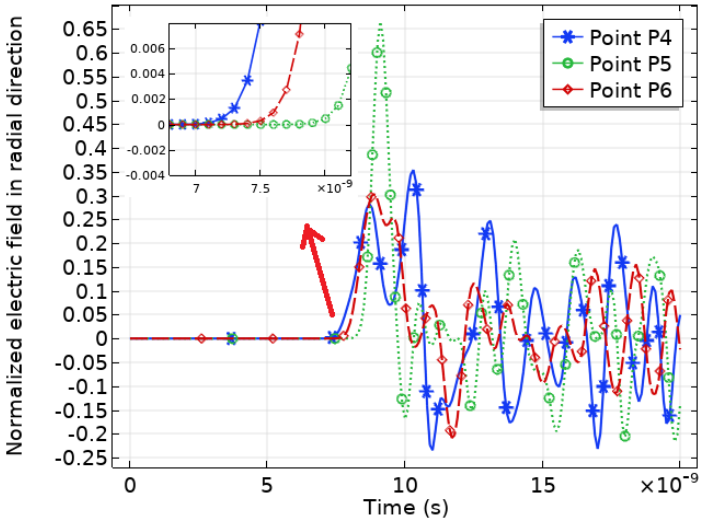

(b)

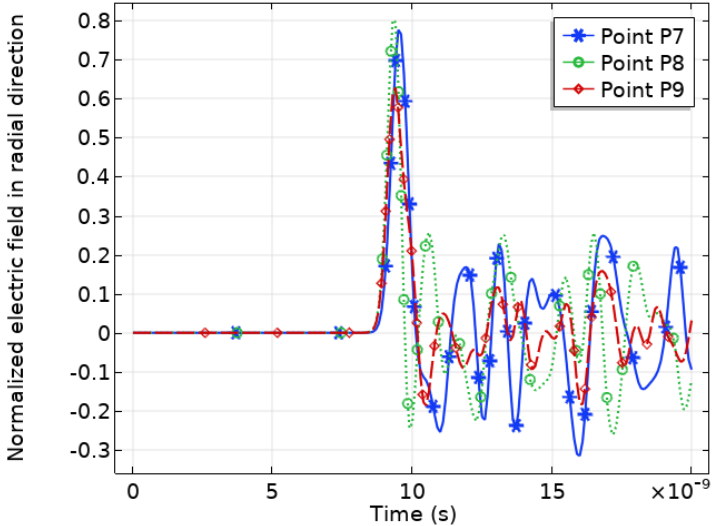

(c)

Fig. 13. Comparison of electric field at point probes (the maximum on point P3 is as the normalized benchmark). (a) Point probes in front of the T-bend. (b) Point probes in tangential turning direction. (c) Point probes in forward direction. 
TABLE III

ATTENUATION AND TIME DELAY EFFECT OF T-BEND (CASE II)

\begin{tabular}{l|c|c|c}
\hline $\begin{array}{c}\text { Point probe } \\
\text { positions }(x, y, z) \\
(\mathrm{mm})\end{array}$ & $\begin{array}{c}\text { Normalized } \\
\text { electric field in } \\
\text { radial direction }\end{array}$ & $\begin{array}{c}\text { TOA based on } \\
\text { signal departure } \\
\text { from zero (ns) }\end{array}$ & $\begin{array}{c}\text { TOA based on } \\
5 \% \text { threshold of } \\
\text { peak (ns) }\end{array}$ \\
\hline P1 $(0,210,500)$ & $99 \%$ & 4.9 & 5.5 \\
P2 $(0,-210,500)$ & $100 \%$ & 4.9 & 5.5 \\
P3 $(-210,0,500)$ & $100 \%$ & 4.9 & 5.5 \\
P4 $(0,500,210)$ & $35 \%$ & 6.8 & 7.8 \\
P5 $(0,500,-210)$ & $67 \%$ & 7.6 & 8.5 \\
P6 $(-210,500,0)$ & $30 \%$ & 7.2 & 8.1 \\
P7 $(0,210,-500)$ & $78 \%$ & 8.2 & 8.9 \\
P8 $(0,-210,-500)$ & $80 \%$ & 8.1 & 8.8 \\
P9 $(-210,0,-500)$ & $63 \%$ & 8.1 & 8.8 \\
\hline
\end{tabular}

\section{The NATURe OF $\mathrm{d} \boldsymbol{E}_{r} / \mathrm{d} t$}

The probe coupler is one kind of sensor used in PD UHF detection technology in GIS. Such couplers do not measure the UHF electric field strength directly and the output voltage response is proportional to $\mathrm{d} \boldsymbol{E}_{\boldsymbol{r}} / \mathrm{d} t$, where $\boldsymbol{E}_{\boldsymbol{r}}$ is the electric field in the radial direction of the measured position [6]. Fig. 14 displays the measured $\mathrm{d} \boldsymbol{E}_{r} / \mathrm{d} t$ in the radial direction at all point probe positions in the L-type structure, and similarly the $\mathrm{d} \boldsymbol{E}_{r} / \mathrm{d} t$ values for the Ttype structure Case I and Case II.

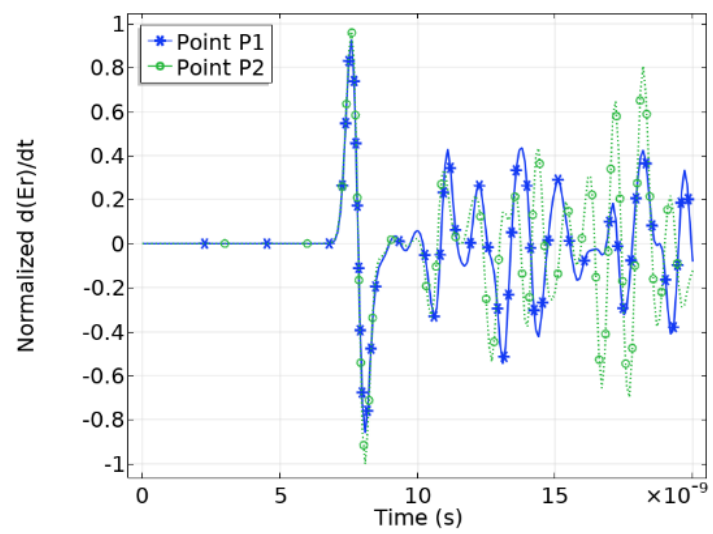

(a)

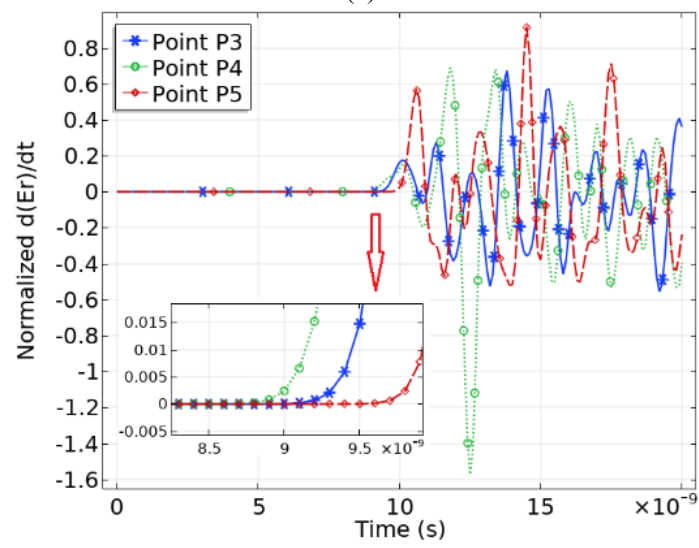

(b)

Fig. 14. Normalized $\mathrm{d} \boldsymbol{E}_{r} / \mathrm{dt}$ at point probes in L-type structure shown in Fig. 7a (the maximum on point P2 is as the normalized benchmark). (a) Point probes on horizontal branch. (b) Point probes on vertical branch.

The attenuation characteristics and time delay for EM wave propagation can be obtained for $\mathrm{d} \boldsymbol{E}_{r} / \mathrm{d} t$ and similar conclusions on attenuation characteristics and time delay also drawn. Notably, the attenuation rate calculated from $\mathrm{d} \boldsymbol{E}_{r} / \mathrm{d} t$ is different from that calculated from $\boldsymbol{E}_{\boldsymbol{r}}$, and in terms of TOA, the results calculated by $\mathrm{d} \boldsymbol{E}_{r} / \mathrm{d} t$ and $\boldsymbol{E}_{\boldsymbol{r}}$ respectively are the same. The specific comparison results are listed in Table IV.

Table IV indicates that the detection sensitivity of $\mathrm{d} \boldsymbol{E}_{r} / \mathrm{d} t$ is higher at the test points in the horizontal plane compared to those positions in the vertical plane of the scheme plan of the chamber. This is consistent with the previous results based on $\boldsymbol{E}_{\boldsymbol{r}}$ detection. It is noteworthy that there seems to be a magnifying effect for $\mathrm{d} \boldsymbol{E}_{r} / \mathrm{d} t$ detection at certain test points and the normalized $\mathrm{d} \boldsymbol{E}_{\boldsymbol{r}} / \mathrm{d} t$ can be larger than $100 \%$. In other words, if $\mathrm{d} \boldsymbol{E}_{r} / \mathrm{d} t$ is normalized to the closest probe, but it is possible for probes that are further away to exceed that value.

In addition, it must be emphasized that the simulation is free from external interference that often takes place on GIS sites. Therefore, besides the distortion of the waveform produced by reflections in the GIS, external noise may have a significant impact on the identification of any field measured TOA.

TABLE IV

ATTENUATION AND TIME DELAY EFFECT ON $\mathrm{d} \boldsymbol{E}_{\boldsymbol{r}} / \mathrm{d} t$

\begin{tabular}{c|l|c|c}
\hline Structures & $\begin{array}{c}\text { Point probe positions } \\
(x, y, z)(\mathrm{mm})\end{array}$ & $\begin{array}{c}\text { Normalized } \\
\mathrm{d} \boldsymbol{E}_{r} / \mathrm{d} t\end{array}$ & $\begin{array}{c}\text { TOA based on 5\% } \\
\text { threshold }(\mathrm{ns})\end{array}$ \\
\hline L-type & P1 $(-210,500,0)$ & $93 \%$ & 7.1 \\
& P2 $(0,500,210)$ & $100 \%$ & 7.1 \\
& P3 $(-210,0,500)$ & $67 \%$ & 9.7 \\
& P4 $(0,210,500)$ & $157 \%$ & 9.4 \\
& P5 $(0,-210,500)$ & $92 \%$ & 10.1 \\
\hline T-type & P1 $(-210,500,0)$ & $99 \%$ & 7.0 \\
Case I & P2 $(0,500,210)$ & $100 \%$ & 7.0 \\
& P3 $(-210,0,500)$ & $64 \%$ & 9.6 \\
& P4 $(0,210,500)$ & $123 \%$ & 9.4 \\
& P5 $(0,-210,500)$ & $90 \%$ & 10.1 \\
\hline T-type & P1 $(0,210,500)$ & $94 \%$ & 5.4 \\
Case II & P2 $(0,-210,500)$ & $100 \%$ & 5.4 \\
& P3 $(-210,0,500)$ & $100 \%$ & 5.4 \\
& P4 $(0,500,210)$ & $59 \%$ & 7.7 \\
& P5 $(0,500,-210)$ & $58 \%$ & 8.3 \\
& P6 $(-210,500,0)$ & $28 \%$ & 7.9 \\
& P7 $(0,210,-500)$ & $74 \%$ & 8.8 \\
& P8 $(0,-210,-500)$ & $118 \%$ & 8.7 \\
& P9 $(-210,0,-500)$ & $58 \%$ & 8.7 \\
\hline
\end{tabular}

\section{DISCUSSION}

The EM wave propagation is influenced by small structural details, such as the spacer shape, conductor shield and so on. Meanwhile, the differences in source input between simulation and real life also influence the test results, like the rise time and waveform of the source pulse. The apparent 'attenuation' effects are due to interference caused by signal energy reflections. These interactions have the potential to impact wave amplitude thresholding processes often used to determine sensor TOA values. Clearly, any impact on TOA evaluations will influence determination of EM wave propagation velocity.

The simulations of L-type structure with shields at the inner conductor are conducted. The profile of the inner conductor is shown in Fig. 15. The electric field at test point A, B, C, and D is illustrated in Fig. 16 and the attenuation and TOA at point probes P1, P2, P3, P4 and P5 near L-bend are shown in Fig. 17 and Table $\mathrm{V}$.

It can be concluded from the results that when compared to the model with no shields, the model with shields produces some signal attenuation. While the detection sensitivity is higher at the test points $\mathrm{P} 4$ and $\mathrm{P} 5$ than that at $\mathrm{P} 3$, which is the same as that in the model without shields. The effect on TOA is 
also the same as that in the model without shields.

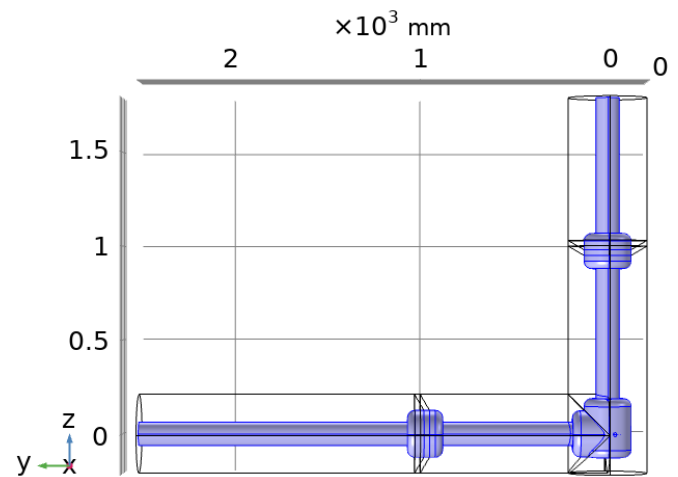

Fig. 15. Geometry of L-type structure and inner conductor with shields 2 times the diameter of the conductor.

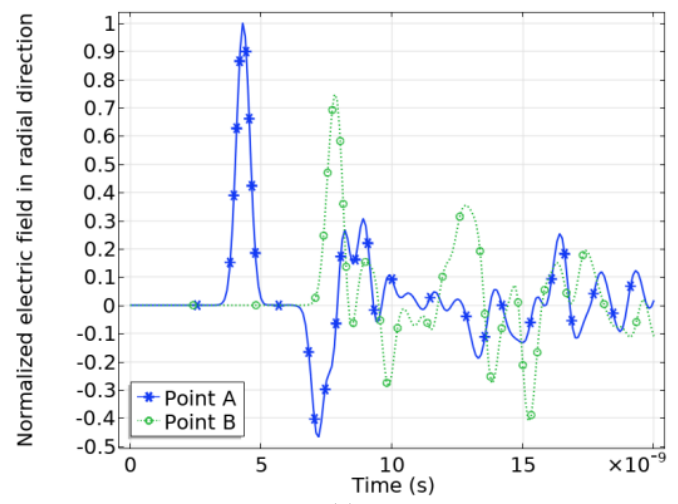

(a)

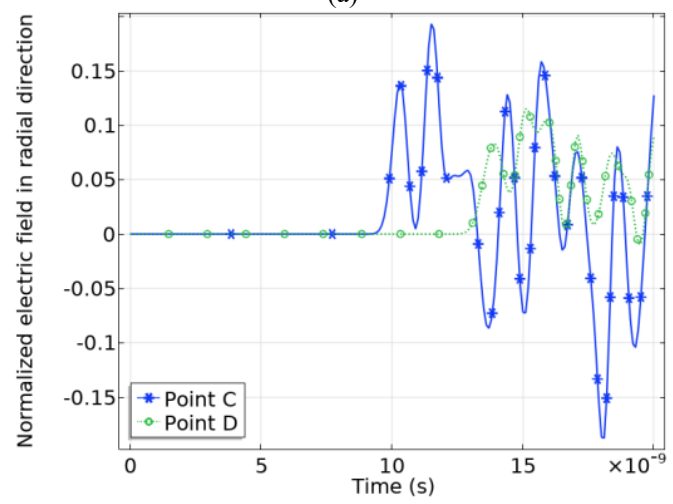

(b)

Fig. 16. Electric field in radial direction at point probes A, B, C, and D (the maximum on point $\mathrm{A}$ is as the normalized field benchmark and the coordinates of point probes are shown in Fig. 4). (a) Point probes A and B on horizontal branch. (b) Point probes $\mathrm{C}$ and $\mathrm{D}$ on vertical branch.

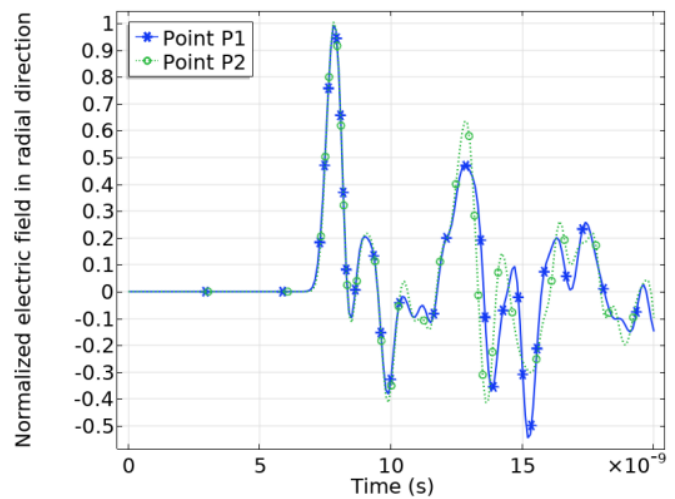

(a)

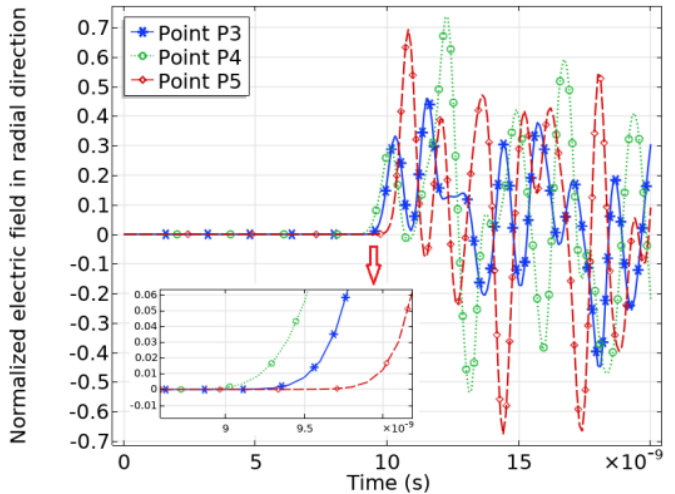

(b)

Fig. 17. Electric field in radial direction at point probes P1, P2, P3, P4 and P5 (the maximum on point $\mathrm{P} 2$ is as the normalized field benchmark and the coordinates of point probes are shown in Fig. 7a). (a) Point probes P1 and P2 on horizontal branch. (b) Point probes P3, P4, and P5 on vertical branch.

TABLE V

ATtENUATION AND TOA AT POINT PROBES P1, P2, P3, P4 AND P5.

\begin{tabular}{c|c|c}
\hline $\begin{array}{c}\text { Point probe positions } \\
(x, y, z)(\mathrm{mm})\end{array}$ & $\begin{array}{c}\text { Normalized electric } \\
\text { field in radial direction }\end{array}$ & $\begin{array}{c}\text { TOA based on 5\% } \\
\text { threshold of peak }(\mathrm{ns})\end{array}$ \\
\hline P1 $(-210,500,0)$ & $99 \%$ & 7.1 \\
P2 $(0,500,210)$ & $100 \%$ & 7.2 \\
P3 $(-210,0,500)$ & $46 \%$ & 9.7 \\
P4 $(0,210,500)$ & $74 \%$ & 9.5 \\
P5 $(0,-210,500)$ & $69 \%$ & 10.2 \\
\hline
\end{tabular}

\section{CONCLUSIONS}

In this study, the attenuation characteristics and time delay produced by barrier spacers, L-type and T-type structures of GIS in EM wave propagation behavior have been investigated. The simulations indicate an overlap of adjacent waves caused by wave reflection so that the time-varying electric field differs quite significantly for different positions at which UHF PD sensors might be mounted. Therefore, appropriate selection of sensor installation positions relative to the L-type and T-type arrangements of GIS bus bars might ensure a more consistent PD detection sensitivity.

It is indicated by comparison that the detection sensitivity is higher at the test points in the horizontal plane compared to those positions in the vertical plane of the cross section of the GIS tank. This result is established both in the calculation by $\boldsymbol{E}_{\boldsymbol{r}}$ and $\mathrm{d} \boldsymbol{E}_{r} / \mathrm{d} t$ respectively and is the same as that in the model with inner conductor shields. The effect on TOA is also the same as that in the model without shields. Therefore, if the location of the electric field detected by the sensor is chosen appropriately, the measurement sensitivity obtained can minimize the effects of attenuation introduced by the L-type and T-type structure.

The effects of time delay produced by barrier spacers, L-type and T-type structures are different from each other. One spacer in this model produces a time delay of approximately $0.1 \mathrm{~ns}$ which accounts for a localization error of approximately $3 \mathrm{~cm}$. Time delay effects caused by the L-type and T-type Case I structures are very similar. The largest difference between TOA of the test point probes after the bend is about $0.9 \mathrm{~ns}$ based on signal zero-crossing and $0.7 \mathrm{~ns}$ based on $5 \%$ peak value, corresponding to a distance of about $27 \mathrm{~cm}$ and $21 \mathrm{~cm}$ respectively for the wave propagation. TOA values will change slowly if another threshold is selected because the amplitude of the waveforms varies over time from place to place and the peak 
values are also different for different test positions.

The time delay effect produced by the T-type Case II structure is different in tangential and forward directions. The arrival time at the points in the tangential direction vary greatly, while the values at the points in the forward direction are essentially the same. Furthermore, the TOA of the points in the forward direction is delayed compared to the tangential branch and the median value of time delay is about $0.9 \mathrm{~ns}$ based on signal zerocrossing and $0.7 \mathrm{~ns}$ based on $5 \%$ peak value.

The attenuation rate calculated from $\mathrm{d} \boldsymbol{E}_{r} / \mathrm{d} t$ is also different from that calculated using $\boldsymbol{E}_{\boldsymbol{r}}$ while, in terms of TOA, the results calculated by $\boldsymbol{E}_{\boldsymbol{r}}$ and $\mathrm{d} \boldsymbol{E}_{\boldsymbol{r}} / \mathrm{d} t$ are the same.

Further validation and application of the conclusions in this paper will be carried out as part of future work.

\section{REFERENCES}

[1] P. Wenger, M. Beltle, S. Tenbohlen, et al, "Combined characterization of free-moving particles in HVDC-GIS using UHF PD, high-speed imaging, and pulse-sequence analysis," IEEE Trans. Power Del., vol. 34, no. 4, pp. 1540-1548, Aug. 2019.

[2] G. Behrmann, W. Koltunowicz and U. Schichler, "State of the art in GIS PD diagnostics," 2018 Condition Monitoring and Diagnosis (CMD), Perth, WA, 2018, pp. 1-6.

[3] T. Hoshino, S. Maruyama, T. Sakakibara, "Simulation of propagating electromagnetic wave due to partial discharge in GIS using FDTD," IEEE Trans. Power Del., vol. 24, no. 1, pp. 153-159, Jan. 2009.

[4] CIGRE WG D1.25, UHF partial discharge detection system for GIS: Application guide for sensitivity verification, CIGRE Tech. Brochure 654, 2016.

[5] W. Gao, D. Ding, W. Liu, and X. Huang, "Propagation attenuation properties of partial discharge in typical in-field gis structures," IEEE Trans. Power Deliv., vol. 28, no. 4, pp. 2540-2549, Oct. 2013.

[6] M. Judd, O. Farish and B. Hampton, "Modelling partial discharge excitation of UHF signals in waveguide structures using Green's functions", IEE Proc. Science, Measurement and Technology, Vol. 143, No. 1, pp. 63-70, Jan. 1996.

[7] M. Yoshimura et al, "Propagation properties of electromagnetic wave through T-branch in GIS,"IEEE Trans. Dielectr. Electr. Insul., vol. 14, no. 2, pp. 328-333, Apr. 2007.

[8] S. Okabe et al, "Partial discharge diagnosis method using electromagnetic wave mode transformation in gas insulated switchgear,"IEEE Trans. Dielectr. Electr. Insul., vol. 14, no. 3, pp. 702-709, Jun. 2007.

[9] S. Okabe et al, "Simulation of propagation characteristics of higher order mode electromagnetic waves in GIS,"IEEE Trans. Dielectr. Electr. Insul., vol. 13, no. 4, pp. 855-861, Aug. 2006.

[10] M. Hikita et al, "Influence of insulating spacer type on propagation properties of PD-induced electromagnetic wave in GIS,"IEEE Trans. Dielectr. Electr. Insul., vol. 17, no. 5, pp. 1642-1648, Oct. 2010.

[11] M. Hikita et al, "Propagation properties of PD-induced electromagnetic wave in $66 \mathrm{kV}$ GIS model tank with L branch structure,"IEEE Trans. Dielectr. Electr. Insul., vol. 18, no. 5, pp. 1678-1685, Oct. 2011.

[12] M. Hikita et al, "Propagation properties of PD-induced electromagnetic wave in GIS model tank with T branch structure," IEEE Trans. Dielectr. Electr. Insul., vol. 18, no. 1, pp. 256-263, Feb. 2011.

[13] M. Hikita et al, "Study of partial discharge radiated electromagnetic wave propagation characteristics in an actual $154 \mathrm{kV}$ model GIS,"IEEE Trans. Dielectr. Electr. Insul., vol. 19, no. 1, pp. 8-17, Feb. 2012.

[14] G. Behrmann et al, "UHF PD signal transmission in GIS: Effects of $90^{\circ}$ bends and an L-shaped CIGRE step 1 test section," IEEE Trans. Dielectr. Electr. Insul., vol. 26, no. 4, pp. 1293-1300, 2019.

[15] G. Behrmann and J. Smajic, "RF PD signal propagation in GIS: Comparing S-parameter measurements with an RF transmission model for a short section of GIS," IEEE Trans. Dielectr. Electr. Insul., vol. 23, no. 3, pp. 1331-1337, 2016.
[16] G. Behrmann et al, "Signal delay effects of solid dielectrics on time-offlight measurements in GIS," IEEE Trans. Dielectr. Electr. Insul., vol. 23, no. 3, pp. 1275-1284, 2016.

[17] T. Zhao, M. D. Judd and B. G. Stewart, "The time dependent simulation of PD electromagnetic wave propagation in GIS systems," IEEE Conf. Electr. Insul. Dielect. Phenom (CEIDP), 2020, pp. 1-4.

[18] M. D. Judd and O. Farish, "High bandwidth measurement of partial discharge current pulses," Proc. IEEE Int. Symp. Elect. Insul., vol. 2, Jun. 1998, pp. 436-439.

[19] M. D. Judd, O. Farish and B. F. Hampton, "The excitation of UHF signals by partial discharges in GIS," IEEE Trans. Dielectr. Electr. Insul., vol. 3, no. 2, pp. 213-228, April 1996.

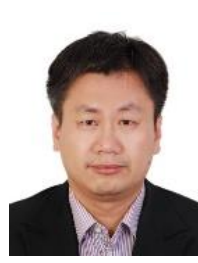

Tao Zhao (M'19) was born in Baoding, Hebei, China in 1982. He received the B.Eng. degree from North China Electric Power University in 2005, the M.Sc. degree from the Chongqing University in 2008 and the $\mathrm{PhD}$ degree from North China Electric Power University in 2017. His research interests include the insulation monitoring of power transformer and fault detection and diagnosis of electric equipment.

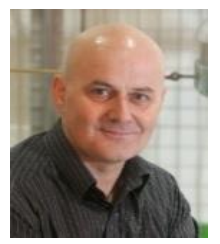

Brian G. Stewart (M'08) is a Professor within the Institute of Energy and Environment at the University of Strathclyde, Glasgow, Scotland. $\mathrm{He}$ graduated with a BSc (Hons) and $\mathrm{PhD}$ from the University of Glasgow in 1981 and 1985 respectively. He also graduated with a BD (Hons) in 1994 from the University of Aberdeen, Scotland. His research interests include high voltage engineering, electrical condition monitoring, insulation diagnostics and communication systems. He is also a Member of the IET (MIET), a Chartered Engineer (CEng) and is currently serving as Vice-President (Administration) of the IEEE DEIS Society.

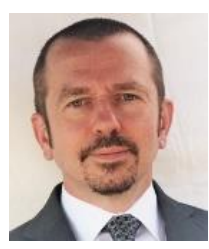

Martin D. Judd (M'02-SM'04) is Technical Director of High Frequency Diagnostics (HFDE) Ltd, based in Scotland. He holds a first class (Hons) degree in Electronic Engineering (University of Hull, 1985) and a PhD (University of Strathclyde, 1996) for research into the excitation of UHF signals by partial discharges in gas insulated switchgear. He has worked extensively on UHF partial discharge monitoring techniques for GIS and transformers and was latterly Professor of High Voltage Technologies at the University of Strathclyde. In 2014, he founded HFDE, a specialist consultancy company that provides diagnostic services and works with businesses developing new monitoring technologies and applications. 